\title{
On a problem of K.A. Bush concerning Hadamard matrices
}

\section{W.D. Wallis}

K.A. Bush has asked whether there is a symmetric Hadamard matrix of order $m^{2}, m$ even, which can be partitioned into an $m \times m$ array of $m \times m$ blocks, such that:

(i) each diagonal block has every entry 1 ;

(ii) each non-diagonal block has every row-sum zero?

We give two ways of constructing such matrices.

1. The problem as posed

We shall assume familiarity with Latin squares, finite projective planes and Hadamard matrices. A suitable reference is [6].

Bush asked the following question* at the conference on Combinatorial Mathematics in Calgary, June, 1969 (see [5, p. 503]):

Given an even integer $m$, is there an Hadamard matrix of the form

$$
H=\left[\begin{array}{cccc}
J & H_{12} & \cdots & H_{1 m} \\
H_{21} & J & \ldots & H_{2 m} \\
& & \cdots & \\
H_{m 1} & H_{m 2} & \cdots & J
\end{array}\right],
$$

where the blocks are of size $m \times m, J$ is the matrix with every entry +1 , and each $H_{i j}$ is a $(1,-1)$-matrix with every row-swm

Received 10 November 1971.

* There is a misprint in [5], but the actual question intended is clear from [3] and [4]. 
and every column-sum zero?

He shows in [4] that the existence of such a matrix is implied by the existence of a finite projective plane of order $m$, and consequently the non-existence of such a matrix of order $m^{2}$ would be of great significance (except for the case $m=6$ ).

We shall give two methods of constructing matrices of the form requested by Bush.

\section{Graphical interpretation of the problem}

We have used the terms design graph and $(v, k, \lambda)$-graph to mean a finite undirected graph on $v$ vertices such that every vertex is adjacent to $k$ others and that any two vertices have $\lambda$ further vertices adjacent to both. (See, for example, [7], [8].)

The Hadamard matrix $H$ of $(1)$ is symetric, has constant row-sum $m$ and has every diagonal entry +1 . Consequently [7, p. 327] it is equivalent to a design graph with parameters $v=4 u^{2}, k=2 u(u-1)$, $\lambda=u(u-1)$, where $m=2 u$; the vertices of the graph correspond to the rows of $H$, and vertices $i$ and $j$ are adjacent if and only if $H$ has $(i, j)$ entry -1 . Therefore Bush's problem can be stated as follows

Given an integer $u$, is there a design graph of parameters $\left(4 u^{2}, 2 u^{2}-u, u^{2}-u\right)$ whose vertices can be partitioned into $2 u$ sets of size $2 u$, such that:

(A) no two vertices in the some set are adjacent;

(B) a vertex in a given set is adjacent to exactly $u$ of the vertices in any other set?

\section{Latin square graphs}

Suppose $L_{1}, L_{2}, \ldots, L_{t}$ are $t$ mutually orthogonal Latin squares of side $s$. We construct a graph whose vertices are the $s^{2}$ ordered pairs $(1,1),(1,2), \ldots,(s, s)$. Two distinct vertices $(a, b)$ and $(c, d)$ are adjacent if and only if

(i) $a=c$, 
(ii) $b=d$, or

(iii) $L_{i}$ has the same entry in positions $(a, b)$ and $(c, d)$ for some $i$.

The graph is called a Latin square graph $L_{t+2}(s)$. It is easy to check that an $L_{u}(2 u)$ graph is a design graph with parameters $\left(4 u^{2}, 2 u^{2}-u, u^{2}-u\right)$ [1].

Assume that $u-1$ mutually orthogonal Latin squares exist of side $2 u$; denote by $\underline{\underline{G}}$ an $L_{u}(2 u)$ graph constructed using $u-2$ of them, and let $L$ be the unused square. Partition the vertices of $\underline{\underline{G}}$ into sets $v_{1}, v_{2}, \ldots, v_{u}$, where $v_{i}$ contains all pairs $(a, b)$ such that the $(a, b)$ entry of $L$ is $i$.

Suppose two members of $V_{i}$ are adjacent in $\underline{G}$. Then either the positions corresponding to them are in the same row or the same column of $L$ (which is impossible, as $i$ occurs once in each row and column of $L$ ), or some other Latin square has the same entry in both the corresponding positions (which is impossible by orthogonality). So no two vertices in the same set are adjacent, that is $\underline{\underline{G}}$ satisfies condition $(A)$.

Suppose $(a, b)$ is a member of $V_{i}$. Of the vertices in $V_{j}$, where $i \neq j,(a, b)$ will be adjacent to one by virtue of (i) since $L$ has entry $j$ once in row $a$, and one by virtue of (ii) similarly. Each Latin square $L_{x}$ other than $L$ will contain the symbols $1,2, \ldots, 2 u$ once in the positions where $L$ has $j$, so $L_{x}$ will have the same symbol in exactly one of these places as it has in position $(a, b)$; this gives $u-2$ further vertices adjacent to $(a, b)$ because of (iii). None of these positions can be in row $a$ or column $b$, and no two of them can be identical (if $L_{x}$ and $L_{y}$ give rise to the same position then $L_{x}$ and $L_{y}$ are not orthogonal). So $(a, b)$ is adjacent to exactly $u$ vertices in $V_{j}$, and $\underline{G}$ satisfies condition $(B)$.

We have proven

THEOREM 1. If there exist $u-1$ mutually orthogonal Latin squares 
of side $2 u$, then there is an Hadamard matrix of type (1) for $m=2 u$.

This result is significant because a projective plane of order $2 u$ is equivalent to $2 u-1$ mutually orthogonal Latin squares of that order. Bruck [2] has shown that slightly less than $2 u-1$ squares are sufficient for a plane, but it is not known that $u-l$ squares are sufficient.

\section{Graphs from Hadamard matrices}

In [8] (see also [9]) we proved the following theorem ([8, Theorem 1]):

LEMMA 1. Suppose there exist an affine resolvable balanced incomplete block design $A R(n, \mu)$ and a balanced incomplete block design with parometers $(v, b, r, k, 1)$, where

$$
r=n \mu+(\mu-1)(n-1)^{-1}+\mu \text {. }
$$

Then there is a strongly regular graph $\underline{\underline{G}}$ with parameters

$$
\left(v n^{2} \mu ;(v-1) n \mu ;(v-k) \mu+(k-2) n \mu,(v-k) \mu\right) ;
$$

moreover the vertices of $\mathrm{G}$ can be partitioned into $v$ subsets $A^{l}, A^{2}, \ldots, A^{v}$ of size $n^{2} \mu$ whose induced subgraphs are nulz.

We now prove

LEMMA 2. In the notation of Lemma 1 , if $x$ is any member of $A^{\beta}$ then for any $\gamma \neq \beta$ there are exactly $n \mu$ vertices in $A^{\gamma}$ which are adjacent to $x$.

Proof. In the interests of brevity we will assume all notations of Theorem 1 of [8].

From the second paragraph of the proof of that theorem, vertices from $A^{\beta}$ and $A^{\gamma}$ are adjacent in exactly one of the subgraphs $\mathrm{G}_{i}$, the one corresponding to the unique block $B_{i}$ containing $\beta$ and $\gamma$, and in $G_{i}$ the vertex $x$ occurs in exactly one subgraph of type $\underline{\underline{K}}_{n \mu}^{k} ;$ in that $\underline{\underline{K}}_{n \mu}^{k}$ it is adjacent to precisely $n \mu$ vertices in $A^{\gamma}$, namely the $n \mu$ vertices in the chosen line of the chosen parallel class $L_{\sigma}^{\gamma}$. Thus $x$ is adjacent 
to precisely $n \mu$ members of $A^{\gamma}$.

THEOREM 2. If there is an Hadamard matrix of order $4 \mu$, then there is an Hadomard matrix of type (1) for $m=4 \mu$.

Proof. An hadamard matrix of order $4 \mu$ is equivalent to an $A R(2, \mu)$ design, as was pointed out in [8]; there is always a balanced incomplete block design with parameters $(4 \mu, 2 \mu(4 \mu-1), 4 \mu-1,2,1)$. So the theorem follows from Lemmas 1 and 2.

The result of Theorem 2 was announced by the author in [10].

\section{References}

[1] R.C. Bose and S.S. Shrikhande, "Graphs in which each pair of vertices is adjacent to the same number $d$ of other vertices", Studia Sci. Math. Hungar. 5 (1970), 181-195.

[2] R.H. Bruck, "Finite nets, II. Uniqueness and imbedding", Pacific $J$. Math. 13 (1963), 421-457.

[3] K.A. Bush, "An inner orthogonality of Hadamard matrices", J. Austral. Math. Soc. 12 (1971), 242-248.

[4] K.A. Bush, "Unbalanced Hadamard matrices and finite projective planes of even order", J. Combinatorial Theory Ser. A 11 (1971), $38-44$.

[5] Richard Guy, Haim Hanani, Norbert Sauer and Johanan Schönheim, (Editors), Combinatorial structures and their applications. Proc. Calgary Internat. Conf. Univ. Calgary, Calgary, Alberta, 1969. (Gordon and Breach, New York, London, Paris, 1970).

[6] Marshall Hall, Jr, Combinatorial theory (Blaisdell Publishing Co. [Ginn and Co.], Waltham, Massachusetts; Toronto, Ontario; London; 1967).

[7] W.D. Wallis, "Certain graphs arising from Hadamard matrices", Bulz. Austral. Math. Soc. 1 (1969), 325-331.

[8] W.D. Wallis, "Construction of strongly regular graphs using affine designs", Buzl. Austral. Math. Soc. 4 (1971), 41-49. 
[9] W.D. Wallis, "Construction of strongly regular graphs using affine designs: Corrigenda", Bulz. Austral. Math. Soc. 5 (1971), 431.

[10] W.D. Wallis, "Special Hadamard matrices", Abstract, to appear in Notices Amer. Math. Soc. 19 (1972).

The University of Newcastle,

Newcastle,

New South Wales. 